\title{
Consumo de Álcool nos Estudantes do Ensino Superior de Coimbra e o Impacto das Festas Académicas
}

\section{Alcohol Drinking in Higher Education Students from Coimbra and the Impact of Academic Festivities}

\author{
André COIMBRA TRIGO $\square^{1}$, Luiz Miguel SANTIAGO 2,3
}

Acta Med Port 2022 Apr;35(4):249-256 - https://doi.org/10.20344/amp.12366

\section{RESUMO}

Introdução: Na literatura internacional, nacional e em estudos no ensino superior em Portugal os jovens apresentam consumos alcoólicos excessivos e binge drinking. Contudo, não se encontra estabelecida qualquer relação entre o consumo e as festas académicas. O objectivo deste estudo foi compreender a associação entre consumo de bebidas alcoólicas e festas académicas, estabelecendo uma relação entre estes momentos e um padrão específico de consumo, bem como perceber a ocorrência de padrões específicos de consumo de acordo com o sexo e os anos de frequência escolar.

Material e Métodos: Foi elaborado um questionário, constituído pelo Alcohol Use Disorders Identification Test - Consumption (AUDIT-C) e apresentando a questão "Qual a bebida mais consumida?" num mês com festas académicas e num mês sem que se realizassem. O questionário dirigido a alunos da Universidade de Coimbra e do Instituto Politécnico de Coimbra, foi divulgado na página de Facebook ${ }^{\circledast}$ da Universidade de Coimbra.

Resultados: $\mathrm{Na}$ amostra de estudantes do ensino superior de Coimbra, com idade igual ou superior a dezassete anos ( $\mathrm{n}=503$ ), o AUDIT-C em mês com festas académicas é superior ao mês sem festas, verificando-se correlação positiva forte e significativa $(\rho=$ $0,711 ; \rho<0,001)$. Em mês sem festas académicas, $41,8 \%$ do sexo feminino e $24,0 \%$ do sexo masculino apresentam AUDIT-C positivo. Em mês com festas académicas $73,8 \%$ do sexo feminino e $56,3 \%$ do sexo masculino apresentam AUDIT-C positivo. O sexo feminino apresenta maior risco de consumo excessivo $(\rho<0,001)$ e bebe mais bebidas espirituosas / shots. Há diferença significativa entre sexos quanto à bebida mais consumida $(\rho<0,001)$.

Conclusão: As festas académicas fomentam as ocasiões de binge drinking pré-existentes, residindo o problema na forma como os jovens abordam o consumo de álcool.

Palavras-chave: Bebedeira; Bebidas Alcoólicas; Comportamento Ritualístico; Educação de Pós-Graduação; Intoxicação Alcoólica

\section{ABSTRACT}

Introduction: The international and national literature as well as studies carried out in universities in Portugal show there is excessive alcohol consumption and binge drinking. However, no relation with alcohol consumption and college parties has yet been established. The aim of this study was to assess the association between alcohol consumption and binge drinking with college parties, and also to understand specific patterns of alcohol consumption by gender and years of university attendance.

Material and Methods: A survey comprised of the Alcohol Use Disorders Identification Test - Consumption (AUDIT-C) and the question "What is your most consumed drink?" referring to months without and with college parties, was disseminated in the University of Coimbra Facebook ${ }^{\circledast}$ page to students of the University of Coimbra and of the Polytechnic Institute of Coimbra seventeen years old and over.

Results: The AUDIT-C score in college party months is higher than in months without such parties with strong, positive and significant correlation $(\rho=0.711 ; \rho<0.001)$. In months without college parties, $41.8 \%$ of females and $24.0 \%$ of males have a positive AUDIT-C. In months with college parties, $73.8 \%$ of females and $56.3 \%$ of males have a positive AUDIT-C. In months with college parties, females have higher risk of excessive consumption $(\rho<0.001)$ and drink a higher amount of spirits / shots. There is a significant difference in the most consumed drinks between genders $(\rho<0.001)$.

Conclusion: College parties allow for more opportunities of pre-existing binge drinking. The way young people face alcohol consumption is a major problem to be addressed.

Keywords: Alcohol Drinks; Alcoholic Intoxication; Binge Drinking; Ceremonial Behavior; Graduate Education

\section{INTRODUÇÃO}

O álcool é uma droga legal, social e culturalmente aceite, causadora de dependência e tem um impacto negativo na saúde quando consumida em excesso. Saber beber moderadamente é um importante fator de convivência e até de integração social. ${ }^{1} \mathrm{O}$ padrão de consumo episódico excessivo, também designado binge drinking, corresponde ao consumo igual ou superior a seis bebidas padrão no homem e cinco bebidas padrão na mulher, numa só ocasião, no espaço de duas horas. ${ }^{2}$
Em 2016, 32,5\% da população mundial consumia bebidas alcoólicas, sendo este comportamento mais observado no sexo masculino. Por sua vez, o sexo feminino bebia menos e exibia menos frequentemente situações de consumo episódico excessivo. ${ }^{3,4}$

O consumo excessivo de bebidas alcoólicas foi apontado como o sétimo maior fator de risco para morte prematura e incapacidade, constituindo o principal fator de risco entre a população mais jovem e afetando a saúde,

1. Estudante. Faculdade de Medicina. Universidade de Coimbra. Coimbra. Portugal.

2. Clínica Universitária de Medicina Geral e Familiar. Faculdade de Medicina. Universidade de Coimbra. Coimbra. Portugal.

3. Centro de Estudos e Investigação em Saúde da Universidade de Coimbra. Coimbra. Portugal.

$\square$ Autor correspondente: André Coimbra Trigo. andre-497@hotmail.com

Recebido/Received: 26/05/2019 - Aceite/Accepted: 28/04/2020 - Publicado Online/Published Online: 29/03/2021 - Publicado/Published: 01/04/2022 Copyright @ Ordem dos Médicos 2022 
conforme o tipo e o volume de bebidas consumidas com efeitos orgânicos adversos a longo prazo.

A intoxicação aguda provoca lesões e alterações psicofisiológicas e a dependência pode provocar comportamento auto-lesivo e violência. ${ }^{3}$

O consumo de bebidas alcoólicas é apontado como o fator responsável por $13,5 \%$ das mortes entre os $20-39$ anos. O maior impacto nestas idades tem como consequência o decréscimo da esperança média de vida, por provocar lesões que mais tarde se relacionarão com a causa de morte. ${ }^{4}$

$\mathrm{Na}$ Europa, tem-se verificado o aumento do consumo de cerveja, a bebida mais consumida (40\%), e vinho $(29,8 \%)$. Em oposição, tem vindo a diminuir o consumo de bebidas espirituosas $(27,2 \%){ }^{4}$

A nível global, a população entre 15 - 19 anos mimetiza a população total, e os jovens entre os 20 - 24 anos são consumidores tão ou mais frequentes do que a população geral, o que pode indiciar um pico de prevalência de consumo nestas idades. Quanto ao padrão de consumo, o consumo episódico excessivo é prevalente entre os 15 - 19 anos ( $\geq 20 \%$ ), atingindo o pico entre os $20-24$ anos. ${ }^{4}$ Entre os que bebem, o consumo episódico excessivo é igualmente superior nestas faixas etárias, o que significa que, quando são consumidores, os jovens bebem em sessões de binge drinking. ${ }^{4}$

Em Portugal, o consumo de álcool é superior à média Europeia. ${ }^{4} \mathrm{Na}$ faixa etária entre os 15 e os 24 anos, tem-se verificado um aumento da dependência e do risco de consumo elevado. Em 2016, 83,7\% dos jovens consumiu álcool, não se tendo verificado diferenças entre sexos. Destes, $49,6 \%$ consumiu em binge (diferença de 12,4\% entre o sexo masculino e feminino) e 31,4\% apresentou embriaguez (diferença de $9,5 \%$ entre o sexo masculino e feminino). ${ }^{5}$ Nos últimos anos, tem-se registado uma alteração do perfil de consumo entre os dois sexos, com uma tendência para o aumento de consumo no sexo feminino. ${ }^{6}$

Os dados existentes para a avaliação dos índices de consumo por idade, sexo e instituições de ensino superior (ES) em Portugal são insuficientes, sendo a investigação de grande importância já que o consumo excessivo de álcool é dos fatores que mais pode prejudicar o desenvolvimento do estudante, que enfrenta novas exigências académicas, sociais e desenvolvimentais..$^{3,7-9} \mathrm{~A}$ necessidade de adaptação e de crescimento pessoal está muitas vezes relacionada com a procura e experimentação, que podem conduzir à adoção de comportamentos disfuncionais e estilos de vida menos saudáveis, comprometendo a saúde e o sucesso académico do jovem adulto. ${ }^{8}$

O consumo de bebidas alcoólicas tornou-se moda, e é algo comum entre os jovens nas suas atividades de integração no ES, sendo associado às noites de festa com amigos, ${ }^{9}$ situações em que os jovens procuram os efeitos da embriaguez. ${ }^{10}$ Estudos realizados no ES em Portugal sugerem consumos alcoólicos elevados e um padrão de consumo de binge drinking..$^{1,10-13}$ Contudo, não foi ainda estabelecida qualquer relação entre o consumo de álcool nos meses com festas académicas e o consumo de álcool nos meses sem este tipo de eventos.

Em Coimbra, são vários os momentos de integração dos novos estudantes, destacando-se as principais festas académicas como a Festa das Latas e a Queima das Fitas, pelo que é da maior importância perceber qual o seu real impacto nos padrões de consumo dos jovens estudantes.

Para muitos estudantes, "festas sem álcool são chatas", não acreditando ser "possível organizar uma festa sem bebidas alcoólicas" e afirmam que "beber é uma das formas mais agradáveis de festejar". ${ }^{10} \mathrm{O}$ incentivo ao consumo, pelos pares, é também uma realidade, sobretudo entre o sexo masculino. ${ }^{10}$ Assim, acredita-se que as festas académicas proporcionam um ambiente propício ao consumo excessivo de álcool, representando um risco para os jovens que nelas participam. ${ }^{14}$

Estudos mostraram grande abertura às perguntas do teste de rastreio Alcohol Use Disorders Identification TestConsumption (AUDIT-C), independentemente da quantidade de álcool consumida, o que permitiu uma identificação dos tipos de consumo. ${ }^{15} \mathrm{O}$ rastreio é fundamental, já que foi demonstrado que os consumidores excessivos reduzem os níveis de consumo após serem identificados e alvo de intervenções breves ou plano de tratamento.

O plano de ação é tanto mais eficaz quanto mais cedo for identificado o consumo excessivo. ${ }^{15}$

Dado o impacto noticioso de eventuais excessos que ocorrem durante as festas académicas, o objetivo principal deste estudo foi compreender a associação entre as diversas festas académicas - para integração de novos estudantes - e os níveis médios de consumo de álcool, tentando perceber se existe uma relação entre estes momentos e o padrão específico médio de consumo fora do contexto das festas académicas. Pretendeu-se igualmente perceber se o consumo de bebidas alcoólicas é transversal ou ocasional, e qual o impacto das festas académicas.

Como objetivos secundários, pretendeu-se compreender como é que o tempo de frequência universitário influi no consumo de álcool, qual o tipo de bebida alcoólica mais consumida, e se este varia com as festividades académicas.

\section{MATERIAL E MÉTODOS}

Estudo observacional transversal na população estudantil de conveniência, proveniente do ES de Coimbra, maior de 17 anos, aprovado pela Comissão de Ética da Faculdade de Medicina da Universidade de Coimbra.

Para o estudo foi elaborado um questionário constituído por três segmentos respondidos no mesmo momento, aplicado pela tecnologia Google Formulários.

Foi feito um pré-teste presencial a 32 voluntários para cálculo do tempo de preenchimento e de problemas de compreensibilidade e de agradabilidade. O tamanho da amostra foi determinado para uma população de 35272 estudantes, um intervalo de confiança de $95 \%$ e uma margem de erro de $5 \%$.

Posteriormente, o questionário foi divulgado pela página 
de Facebook ${ }^{\circledR}$ da UC entre 25 de outubro e 20 de novembro de 2018, com dois lembretes eletrónicos. Para garantir a precisão e rigor dos resultados, os participantes foram informados sobre o carácter anónimo, confidencial, e voluntário, do preenchimento do questionário. Assim, foi apresentado um consentimento informado inicial de preenchimento obrigatório para todos os candidatos à participação no estudo. Por motivos práticos e para eliminar duplicações, foram solicitados os três últimos algarismos do Cartão de Cidadão, e a primeira e última letras do apelido dos participantes.

O questionário elaborado tem por base a versão portuguesa do inquérito Alcohol Use Disorders Identification Test - Consumption (AUDIT-C) [Apêndice 1 (ver Apêndice 1: https://www.actamedicaportuguesa.com/revista/index. php/amp/article/view/12366/Apendice_01.pdf)], uma versão reduzida do questionário AUDIT, tendo a versão portuguesa sido validada por Jorge Roque da Cunha em $2002 .{ }^{16}$ O AUDIT-C identifica indivíduos em risco de consumo excessivo de álcool, ${ }^{2}$ utilizando três questões: "Com que frequência consome bebidas que contêm álcool?", "Quando bebe, quantas bebidas contendo álcool consome num dia normal?" e "Com que frequência consome 6 bebidas ou mais numa única ocasião?". Cada questão tem cinco respostas às quais é atribuída uma pontuação de 0 a 4 . Quando a soma das pontuações é igual ou superior a cinco nos homens, e igual ou superior a quatro nas mulheres, estamos perante consumo excessivo de álcool. A pontuação total varia de 0 a 12 pontos.

Assim, o primeiro segmento do questionário apresentou a questão "É estudante do ensino superior em Coimbra?" e um inquérito epidemiológico inicial [Apêndice 1 (ver Apêndice 1: https://www.actamedicaportuguesa.com/revista/ index.php/amp/article/view/12366/Apendice_01.pdf)] com solicitação da idade, sexo e ano de entrada no ES.

O segundo segmento do questionário, reportando-se a um mês sem festas académicas (MSFA), apresentou o o inquérito AUDIT-Ce a questão "Qual a bebida mais consumida [Apêndice 1 (ver Apêndice 1: https://www.actamedicaportuguesa.com/revista/index.php/amp/article/view/12366/ Apendice_01.pdf)].

O terceiro segmento do questionário, reportando-se a um mês com festas académicas (MCFA), apresentou oAUDIT-C, a questão "Qual a bebida mais consumida?" [Apêndice 1 (ver Apêndice 1: https://www.actamedicaportuguesa.com/ revista/index.php/amp/article/view/12366/Apendice_01. pdf)] e a questão "Julga ter o mesmo padrão aproximado de consumo que no resto do tempo?". A questão "Qual a bebida mais consumida?" apresentava três respostas possíveis: cerveja, vinho e outras (shots, bebidas espirituosas...).

Posteriormente, os questionários dos inquiridos que responderam "não ser estudantes" do ES foram descartados. Procedemos à divisão das respostas obtidas em dois grupos, em função dos anos de frequência do ensino superior em até três e superior a três, dado ser este o número de anos para a Licenciatura na esmagadora maioria dos cursos, sendo depois iniciado o mestrado integrado faculta- tivo.

A análise estatística foi efetuada com recurso ao software IBM SPSS Statistics, versão 24.

As variáveis qualitativas e as quantitativas foram descritas pelas frequências absolutas e relativas. Para avaliar a associação entre variáveis qualitativas nominais aplicou-se o teste do Qui-quadrado $\left(\chi^{2}\right)$ para a independência e a relação entre variáveis ordinais não nominais de dois grupos foi analisada pelo teste $U$ de Mann-Whitney, bem como pela correlação de Spearman, por não se ter verificado distribuição normal das variáveis numéricas. O nível de significância adotado no estudo foi 0,01. Calcularam-se ainda dinâmicas de crescimento $(\Delta)$.

\section{RESULTADOS \\ Caracterização demográfica da amostra}

No pré-teste percebeu-se ser de três minutos o tempo de preenchimento, não sendo registadas dificuldades de perceção ou de desagrado perante o questionário.

Obtiveram-se, no total, 518 respostas ao questionário, $503(97,1 \%)$ das quais foram consideradas válidas para estudo por terem preenchido todos os campos necessários e confirmarem ser estudantes do ES de Coimbra. O cálculo da amostra apontava para um valor de 381 .

A idade média foi de $21,8 \pm 3,1$ anos (IC a $95 \%$ de 21,5 a 22,0 anos), variando de 17 a 43 anos.

Dos inquiridos, $148(29,4 \%)$ eram do sexo masculino, a média de anos de frequência do ES era de 3,2 $\pm 2,1$ anos e $239(46,1 \%)$ frequentavam o ES há menos de quatro anos.

Através do teste de Kolmogorov-Smirnov (KS) de uma amostra concluiu-se que as variáveis idade $(p<0,001)$ e anos de frequência do ES em Coimbra $(p<0,001)$ não tinham distribuição normal.

Pelo teste $U$ de Mann-Whitney não se verificaram diferenças na idade entre sexos ( $\delta 22,1 \pm 3,3$ e $q 21,6 \pm 3,0$, $p=0,053)$, nem nos anos de frequência de ES em Coimbra (đ $3,5 \pm 2,4$ e $q 3,1 \pm 2,0, p=0,195)$.

\section{Comparação do padrão de consumo entre MSFA e MCFA}

As variáveis AUDIT-C em MSFA e MCFA não apresentaram uma distribuição normal (KS, $p<0,001)$.

Os consumos são superiores em meses com festas académicas (AUDIT-C em MCFA 5,9 $\pm 3,4$ ) em relação aos meses sem festas académicas (AUDIT-C em MSFA 3,2 \pm 2,3) (Tabela 1). Verifica-se correlação positiva forte e significativa entre os dois valores $(\rho=0,711 ; p<0,001)$, o que indica que aqueles que já apresentavam um maior consumo em meses sem festas terão um maior consumo em meses com festas académicas.

Conforme descrito na Tabela 2, 65,8\% responderam afirmativamente quanto a terem um padrão de consumo diferente num MSFA e num MCFA, não se verificando diferença entre sexos $(p=0,248)$. Já num MSFA, a diferença é significativa, com $41,8 \%$ do sexo feminino e $24,0 \%$ do sexo masculino a apresentarem um AUDIT-C positivo. Também importantes são as diferenças entre sexos em 
Tabela 1 - AUDIT-C em mês sem festas e em mês com festas

\begin{tabular}{ccccc} 
& $\mathbf{n}$ & Média \pm dp & IC a $95 \%$ & $\boldsymbol{\rho} ; \boldsymbol{p}\left(^{*}\right)$ \\
\hline AUDIT-C MSFA & $489^{* *}$ & $3,2 \pm 2,3$ & 3,0 a 3,4 & \\
AUDIT-C MCFA & $489^{* *}$ & $5,9 \pm 3,4$ & 5,7 a 6,2 & 0,$711 ;<0,001$ \\
\hline
\end{tabular}

$\left(^{*}\right)$ Correlação de Spearman;

** Nota do autor: 14 casos omissos $(2,8 \%)$.

MSFA: mês sem festas académicas; MCFA: mês com festas académicas

Tabela 2 - Padrão de consumo e AUDIT-C em MSFA e MCFA, por sexos

\begin{tabular}{|c|c|c|c|c|c|}
\hline & & $\begin{array}{c}\text { Feminino } \\
\mathrm{n}(\%)\end{array}$ & $\begin{array}{c}\text { Masculino } \\
\mathrm{n}(\%)\end{array}$ & $\begin{array}{l}\text { Total } \\
\mathrm{n}(\%)\end{array}$ & $p$ \\
\hline \multirow{2}{*}{$\begin{array}{c}\text { Igual padrão de } \\
\text { consumo em } \\
\text { MSFA e em MCFA? }\end{array}$} & Sim & $127(35,8)$ & $45(30,4)$ & $172(34,2)$ & \multirow{2}{*}{$0,248\left(^{*}\right)$} \\
\hline & Não & $228(64,2)$ & $103(69,6)$ & $331(65,8)$ & \\
\hline \multirow{2}{*}{ MSFA } & AUDIT-C Negativo & $202(58,2)$ & $108(76,0)$ & $310(63,4)$ & \multirow{2}{*}{$\left.<0,001{ }^{(* *}\right)$} \\
\hline & AUDIT-C Positivo & $145(41,8)$ & $34(24,0)$ & $179(36,6)$ & \\
\hline \multirow{2}{*}{ MCFA } & AUDIT-C Negativo & $91(26,2)$ & $62(43,7)$ & $153(31,3)$ & \multirow{2}{*}{$<0,001\left(^{* *}\right)$} \\
\hline & AUDIT-C Positivo & $256(73,8)$ & $80(56,3)$ & $336(68,7)$ & \\
\hline
\end{tabular}

(*) U de Mann-Whitney; $\left.{ }^{(* *}\right) \chi^{2}$

Nota do autor: 14 casos omissos $(2,8 \%)$.

MSFA: mês sem festas académicas; MCFA: mês com festas académicas

Tabela 3 - AUDIT-C, por grupos de anos de frequência do ensino superior e sexo

\begin{tabular}{|c|c|c|c|c|c|c|}
\hline & \multicolumn{2}{|c|}{ Grupos de anos de frequência do Ensino Superior } & \multirow{2}{*}{$\boldsymbol{p}\left({ }^{*}\right)$} & \multicolumn{2}{|c|}{ Sexo } & \multirow{2}{*}{$p\left(^{*}\right)$} \\
\hline & Até 3 inclusive & Superior a 3 & & Feminino & Masculino & \\
\hline AUDIT-C em MSFA & $3,5 \pm 2,3$ & $2,8 \pm 2,3$ & 0,002 & $3,2 \pm 2,4$ & $3,1 \pm 2,3$ & 0,680 \\
\hline AUDIT-C em MCFA & $6,2 \pm 3,4$ & $5,4 \pm 3,3$ & 0,013 & $6,0 \pm 3,4$ & $5,6 \pm 3,2$ & 0,216 \\
\hline
\end{tabular}

$(*) \chi^{2}$

MSFA: mês sem festas académicas; MCFA: mês com festas académicas

MCFA: $73,8 \%$ do sexo feminino e $56,3 \%$ do sexo masculino apresentaram AUDIT-C positivo $(p<0,001)$, sendo a percentagem de AUDIT-C positivos superior em MSFA e MCFA no sexo feminino.

$\mathrm{Na}$ análise de MSFA e MCFA (Tabela 3) realizada em função de Grupo de frequência do ES (até ou superior a três anos) verificou-se diferença apenas em MSFA ( $p=$ 0,002 ), sendo mais elevado o valor nos estudantes com frequência até três anos.

$O$ valor do AUDIT-C foi significativamente superior no grupo até três anos de ES em MSFA $(p=0,002)$, o que não se verificou em MCFA $(p=0,013)$. Registou-se dinâmica de crescimento $(\Delta)$ do AUDIT-C de $+0,77$ e de $+1,35$ entre MSFA e MCFA para o sexo feminino e o masculino, respetivamente.

Realizámos correlação entre a idade dos respondentes e o valor do AUDIT-C, que revelou, em ambiente MSFA, correlação negativa fraca e não significativa $(\rho=-0,082$; $p=0,072)$ e correlação negativa fraca, mas significativa, para o número de anos de frequência de $\mathrm{ES}\left(r_{s}=-0,130 ; p\right.$ $=0,004)$.

Para MCFA, verificou-se correlação negativa fraca e não significativa entre a idade dos respondentes e o valor do AUDIT-C $(\rho=-0,057 ; p=0,205)$ e correlação negativa fraca não significativa entre o valor de AUDIT-C e os anos de frequência do ES $(\rho=-0,104 ; p=0,021)$.

Em MSFA, verificou-se diferença significativa entre sexos quanto à frequência do consumo (pergunta 1 do $\mathrm{AU}$ -
DIT-C) $(p<0,001)$, quantidade (pergunta 2 do AUDIT-C) ( $p$ $<0,001$ ) e frequência de consumo elevado (pergunta 3 do AUDIT-C) $(p<0,001)$, superiores no sexo masculino (Tabela 4).

Já em ambiente MCFA, foi notória uma diferença significativa entre sexos quanto à frequência do consumo (pergunta 1 do AUDIT-C) $(p<0,001)$, quantidade (pergunta 2 do AUDIT-C) $(p<0,001)$ e frequência de consumo elevado (pergunta 3 do AUDIT-C) $(p<0,001)$, superiores no sexo masculino (Tabela 4).

A análise das bebidas mais consumidas é apresentada na Tabela 5. Em MSFA, 55,9\% dos inquiridos consome mais cerveja, $27,8 \%$ outras (shots, bebidas espirituosas) e $16,3 \%$ vinho, não havendo diferença significativa entre grupos de anos de frequência do ES quanto à bebida mais consumida $(p=0,119)$. Contudo, obtiveram-se resultados significativamente diferentes entre sexos na pergunta sobre o tipo de bebida mais consumido $(p<0,001)$.

Quanto às bebidas mais consumidas em MCFA, 52,5\% dos inquiridos consome mais cerveja, $32 \%$ outras (shots, bebidas espirituosas) e $15,5 \%$ vinho. O consumo entre grupos de anos de frequência do ES não revelou diferenças quanto à bebida mais consumida $(p=0,029)$, apesar de entre sexos o comportamento já ser diferente $(p<0,001)$ Tabela 5.

Entre MSFA e MCFA, o valor o tipo de bebida mais consumida teve $\Delta$ de $+0,15$ para bebidas espirituosas/shots sendo $\Delta$ para vinho e cerveja de $-0,04$ e $-0,02$ 
Tabela 4 - Respostas às perguntas do AUDIT-C por sexos e em função de MSFA e MCFA

\begin{tabular}{|c|c|c|c|c|c|}
\hline & & $\begin{array}{c}\text { Feminino } \\
\mathrm{n}(\%)\end{array}$ & $\begin{array}{c}\text { Masculino } \\
\mathrm{n}(\%)\end{array}$ & Total & $\boldsymbol{p}\left({ }^{*}\right)$ \\
\hline \multirow{5}{*}{$\begin{array}{l}\text { Pergunta 1: } \\
\text { AUDIT-C em MSFA }\end{array}$} & Nunca & $49(13,8)$ & $4(2,7)$ & $53(10,5)$ & \multirow{5}{*}{$<0,001$} \\
\hline & 1 vez por mês ou menos & $149(42,0)$ & $32(21,6)$ & $181(36,0)$ & \\
\hline & 2 a 4 vezes por mês & $131(36,9)$ & $67(45,3)$ & $198(39,4)$ & \\
\hline & 2 a 3 vezes por semana & $22(6,2)$ & $38(25,7)$ & $60(11,9)$ & \\
\hline & Mais de 4 por semana & $4(1,1)$ & $7(4,7)$ & $11(2,2)$ & \\
\hline \multirow{5}{*}{$\begin{array}{l}\text { Pergunta 2: } \\
\text { AUDIT-C em MSFA }\end{array}$} & 1 ou 2 & $231(65,1)$ & $70(47,3)$ & $301(59,8)$ & \multirow{5}{*}{$<0,001$} \\
\hline & 3 ou 4 & $62(17,5)$ & $36(24,3)$ & $98(19,5)$ & \\
\hline & 5 ou 6 & $44(12,4)$ & $20(13,5)$ & $64(12,7)$ & \\
\hline & 7 ou 9 & $12(3,4)$ & $9(6,1)$ & $21(4,2)$ & \\
\hline & 10 ou mais & $6(1,7)$ & $13(8,8)$ & $19(3,8)$ & \\
\hline \multirow{5}{*}{$\begin{array}{l}\text { Pergunta 3: } \\
\text { AUDIT-C em MSFA }\end{array}$} & Nunca & $147(41,4)$ & $30(20,3)$ & $177(35,2)$ & \multirow{5}{*}{$<0,001$} \\
\hline & 1 vez por mês ou menos & $162(45,6)$ & $66(44,6)$ & $228(45,3)$ & \\
\hline & 2 a 4 vezes por mês & $39(11,0)$ & $42(28,4)$ & $81(16,1)$ & \\
\hline & 2 a 3 vezes por semana & $3(0,8)$ & $7(4,7)$ & $10(2,0)$ & \\
\hline & Mais de 4 por semana & $4(1,1)$ & $3(2,0)$ & $7(1,4)$ & \\
\hline \multirow{5}{*}{$\begin{array}{l}\text { Pergunta 1: } \\
\text { AUDIT-C em MCFA }\end{array}$} & Nunca & $15(4,2)$ & $3(2,0)$ & $18(3,6)$ & \multirow{5}{*}{$<0,001$} \\
\hline & 1 vez por mês ou menos & $71(20,0)$ & $7(4,7)$ & $161(32,0)$ & \\
\hline & 2 a 4 vezes por mês & $117(33,0)$ & $44(29,7)$ & $161(32,0)$ & \\
\hline & 2 a 3 vezes por semana & $91(25,6)$ & $43(29,1)$ & $134(26,6)$ & \\
\hline & Mais de 4 por semana & $61(17,2)$ & $51(34,5)$ & $112(22,3)$ & \\
\hline \multirow{5}{*}{$\begin{array}{l}\text { Pergunta 2: } \\
\text { AUDIT-C em MCFA }\end{array}$} & 1 ou 2 & $15(4,2)$ & $3(2,0)$ & $18(3,6)$ & \multirow{5}{*}{$<0,001$} \\
\hline & 3 ou 4 & $71(20,0)$ & $7(4,7)$ & $78(15,5)$ & \\
\hline & 5 ou 6 & $117(33,0)$ & $44(29,7)$ & $161(32,0)$ & \\
\hline & 7 ou 9 & $91(25,6)$ & $43(29,1)$ & $134(26,6)$ & \\
\hline & 10 ou mais & $61(17,2)$ & $51(34,5)$ & $112(22,3)$ & \\
\hline \multirow{5}{*}{$\begin{array}{l}\text { Pergunta 3: } \\
\text { AUDIT-C em MCFA }\end{array}$} & Nunca & $97(27,3)$ & $17(11,5)$ & $114(22,7)$ & \multirow{5}{*}{$<0,001$} \\
\hline & 1 vez por mês ou menos & $101(28,5)$ & $28(18,9)$ & $129(25,6)$ & \\
\hline & 2 a 4 vezes por mês & $89(25,1)$ & $41(27,7)$ & $130(25,8)$ & \\
\hline & 2 a 3 vezes por semana & $41(11,5)$ & $27(18,2)$ & $68(13,5)$ & \\
\hline & Mais de 4 por semana & $227(7,6)$ & $35(23,6)$ & $62(12,3)$ & \\
\hline
\end{tabular}

$\left({ }^{*}\right) \cup$ de Mann-Whitney

MSFA: mês sem festas académicas; MCFA: mês com festas académicas

Nota do autor:

Pergunta 1: "Com que frequência consome bebidas que contêm álcool?";

Pergunta 2: "Quando bebe, quantas bebidas contendo álcool consome num dia normal?"; Pergunta 3: "Com que frequência consome 6 bebidas ou mais numa única ocasião?".

respetivamente.

\section{DISCUSSÃO}

A metodologia seguida foi acolhida com facilidade pelos estudantes da Universidade de Coimbra e do Instituto Politécnico de Coimbra, o que permitiu obter respostas mais rápidas de um universo mais abrangente de respondentes, tendo sido alcançado uma volume significativo de respostas a questões relativas ao consumo de álcool.

Os resultados foram obtidos a partir do preenchimento de um questionário Google forms divulgado pela página de Facebook $^{\circledast}$ da UC. Apesar do preenchimento do questionário ser voluntário, a amostra obtida atingiu dimensão representativa.
Tendo em vista uma melhor caracterização e acompanhamento da população, será pertinente, no futuro, disponibilizar o preenchimento do inquérito AUDIT-C a toda a população estudantil em colaboração com as instituições do ES, acrescentando o resultado obtido e respetiva orientação consoante o resultado (se $\geq 5$ no homem e $\geq 4$ na muIher, adicionar perguntas 4 a 10 do AUDIT para classificar $o$ consumo e definir respectiva intervenção).

A maioria das respostas obtidas foram do sexo feminino, o que é concordante com um estudo anteriormente realizado na UC, ${ }^{11,17}$ e com a composição da população estudantil de Coimbra (56,5\% estudantes do sexo feminino). ${ }^{18}$

Contrariamente aos dados nacionais de 2016 do Projeto $\mathrm{HBSC} / \mathrm{JunP},{ }^{19} 65,8 \%$ dos inquiridos reconheceram que 
Tabela 5 - Bebida mais consumida em MSFA e em MCFA, por grupo de anos de frequência do ensino superior e por sexo

\begin{tabular}{|c|c|c|c|c|c|c|}
\hline & & & \multicolumn{3}{|c|}{ Qual a bebida mais consumida } & \multirow[b]{2}{*}{$\boldsymbol{p}\left({ }^{*}\right)$} \\
\hline & & & $\begin{array}{c}\text { Cerveja } \\
\mathrm{n}(\%)\end{array}$ & $\begin{array}{l}\text { Vinho } \\
\mathrm{n}(\%)\end{array}$ & $\begin{array}{c}\text { Outras } \\
\text { (shots, bebidas espirituosas...) } \\
\mathrm{n}(\%)\end{array}$ & \\
\hline \multirow{6}{*}{ MSFA } & & Até três inclusive & $132(55,2)$ & $30(12,6)$ & $77(32,2)$ & \multirow{3}{*}{0,119} \\
\hline & $\begin{array}{l}\text { Grupos de frequência do } \\
\text { Ensino Superior }\end{array}$ & $\begin{array}{c}\text { Igual ou superior } \\
\text { a quatro }\end{array}$ & $136(59,6)$ & $41(18,0)$ & $51(22,4)$ & \\
\hline & & Total & $268(57,4)$ & $71(15,2)$ & $128(27,4)$ & \\
\hline & \multirow{3}{*}{ Sexo } & Feminino & $162(45,6)$ & $63(17,7)$ & $130(36,6)$ & \multirow{3}{*}{$<0,001$} \\
\hline & & Masculino & $119(80,4)$ & $19(12,8)$ & $10(6,8)$ & \\
\hline & & Total & $281(55,9)$ & $82(16,3)$ & $140(27,8)$ & \\
\hline \multirow{6}{*}{ MCFA } & \multirow{3}{*}{$\begin{array}{l}\text { Grupos de frequência do } \\
\text { Ensino Superior }\end{array}$} & Até três inclusive & $120(50,2)$ & $34(14,2)$ & $85(35,6)$ & \multirow{3}{*}{0,029} \\
\hline & & $\begin{array}{c}\text { Igual ou superior } \\
\text { a quatro }\end{array}$ & $136(59,6)$ & $31(13,6)$ & $61(26,8)$ & \\
\hline & & Total & $256(54,8)$ & $65(13,9)$ & $146(31,3)$ & \\
\hline & \multirow{3}{*}{ Sexo } & Feminino & $151(42,5)$ & $63(17,7)$ & $141(39,7)$ & \multirow{3}{*}{$<0,001$} \\
\hline & & Masculino & $113(76,4)$ & $15(10,1)$ & $20(13,5)$ & \\
\hline & & Total & $264(52,5)$ & $78(15,5)$ & $161(32,0)$ & \\
\hline
\end{tabular}

$\left(^{*}\right) \cup$ de Mann-Whitney

Nota de autor: 32 casos omissos $(7,2 \%)$.

MSFA: mês sem festas académicas; MCFA: mês com festas académicas

os consumos são superiores em meses com festas académicas, o que é comprovado com o aumento em cerca de três pontos na média do AUDIT-C $(3,2 \pm 2,3$ em MSFA e 5,9 $\pm 3,4$ em MCFA) e com o aumento da percentagem de AUDIT-C positivos ( $36,6 \%$ em MSFA e $68,7 \%$ em MCFA), que revelam maior consumo excessivo associado às festividades.

A média do AUDIT-C em MSFA encontra-se no limiar do consumo excessivo, com $36,6 \%$ dos inquiridos a registar um AUDIT-C positivo. Este resultado deve ser salientado e alvo de futuros estudos com caráter regular. A média do AUDIT-C em MCFA revela um consumo excessivo para ambos os sexos ( $\geq 5$ no homem, $\geq 4$ na mulher), quase duplicando o número de inquiridos com AUDIT-C positivo $(68,7 \%)$. Para além disto, a forte correlação positiva entre os AUDIT-C em MSFA e MCFA $(\rho=0,711, p<0,001)$ demonstra que quanto mais elevado é o consumo em meses sem festas académicas, maior é o consumo com as festividades.

A idade não apresenta correlação com o consumo de álcool, sendo os nossos resultados coincidentes com outros inquéritos realizados a alunos do ES de Coimbra, Leiria e Aveiro. ${ }^{1}$

O consumo de bebidas alcoólicas parece diminuir ligeiramente com os anos de frequência do ES, quer em MSFA $(\rho=-0,130, p=0,004)$, quer em MCFA $(\rho=-0,104$, $p=0,021)$. Este resultado é corroborado por estudos da Universidade de Aveiro e podem ser explicados pelo facto de os estudantes apresentarem maior necessidade de integração e desinibição nos primeiros anos. ${ }^{13}$ No entanto, a intensificação do consumo com as festas académicas é semelhante em ambos os grupos definidos com base nos anos de frequência (até três anos inclusive, AUDIT-C de 3,5 $\pm 2,3$ para $6,2 \pm 3,4$; mais de três anos, AUDIT-C de $2,8 \pm$ 2,3 para $5,4 \pm 3,3$ ).

Não há diferença significativa entre os sexos, no que diz respeito às médias do AUDIT-C. Este facto poderá ser sugestivo de maior risco de consumo excessivo pelo sexo feminino, tendo em conta que o ponto de corte do AUDIT-C é inferior ( $\geq 4$ no sexo feminino, $\geq 5$ no sexo masculino). De facto, num mês sem festas académicas, $41,8 \%$ do sexo feminino e $24 \%$ do sexo masculino apresentam AUDIT-C positivo $(p<0,001)$. Num mês com festas académicas, os valores sobem significativamente para $73,8 \%$ no sexo feminino e $56,3 \%$ no sexo masculino $(p<0,001)$. Há, portanto, maior percentagem de AUDIT-C positivo no sexo feminino, o que indica maior risco de consumo excessivo entre as estudantes do ES.

Não obstante, em cada pergunta isolada, o sexo masculino tem respostas tendentes a crer-se em maior consumo, exibindo consumo superior ao máximo recomendado e binge drinking mais frequente, à semelhança de outro estudo realizado no ES em Coimbra. ${ }^{11}$

Os presentes resultados divergem dos resultados obtidos em estudos realizados noutros países, ${ }^{20,21}$ e no ES em Portugal, tal como em Aveiro, onde o sexo masculino apresentou médias de consumo superiores. ${ }^{1,7,9,13}$ Tais diferenças poderão dever-se ao tamanho da amostra, distribuição e eventual alteração dos hábitos de consumo desde que foram realizados estes estudos. ${ }^{17,9,13}$ Contudo, o inquérito aos jovens participantes no Dia da Defesa Nacional mostra um padrão de consumo semelhante entre ambos os sexos. Os resultados deste estudo são concordantes com os de estudos mais recentes. ${ }^{5}$

No que diz respeito aos padrões de consumo, a percentagem de abstinentes num MSFA é de $13,8 \%$ no sexo 
feminino e de $2,7 \%$ no sexo masculino, valores inferiores aos dados nacionais, ${ }^{5}$ mas semelhantes aos dos jovens de 18 anos que participaram no Dia da Defesa Nacional. ${ }^{4}$ Estes resultados poderão estar relacionados com a faixa etária deste estudo, que poderá estar a atingir o pico da prevalência de consumo. ${ }^{4}$ Num MCFA, estas percentagens de abstinentes descem para $4,2 \%$ e $2 \%$, respetivamente.

Quanto à quantidade de álcool consumida num dia normal em MSFA, 35\% do sexo feminino e $52,7 \%$ do sexo masculino têm um consumo superior à quantidade máxima diária recomendada (uma bebida padrão na mulher e duas bebidas padrão no homem), ${ }^{2}$ tal como verificado noutros estudos no Ensino Superior em Coimbra. ${ }^{22}$ Num dia normal de MSFA, $1,7 \%$ das mulheres e $8,8 \%$ dos homens consomem 10 ou mais bebidas. Num MCFA, 95,8\% do sexo feminino e $98 \%$ do sexo masculino têm um consumo superior à quantidade máxima diária recomendada. Registou-se ainda que num dia normal, $7,6 \%$ dos inquiridos do sexo feminino e $23,6 \%$ do sexo masculino consomem 10 ou mais bebidas.

O binge drinking, num MSFA, tem uma frequência de $58,6 \%$ na mulher e de $79,7 \%$ no homem, dos quais $12,9 \%$ e $35,1 \%$ respectivamente, mais duas a quatro vezes por mês, e $1,1 \%$ e $2,0 \%$, respectivamente, quatro ou mais vezes por semana, valores que são preocupantes e superiores aos da literatura nacional e internacional. ${ }^{4,5,22}$

O binge drinking aumenta consideravelmente num MCFA, para $72,7 \%$ no sexo feminino e para $88,5 \%$ no sexo masculino, o que representa $44,2 \%$ e $69,5 \%$, respectivamente com consumos superiores entre duas a quatro vezes por mês, e 7,6\% e 23,6\%, respectivamente, com consumos superiores entre quatro ou mais vezes por semana. Este padrão de binge drinking é considerado consumo de risco pela maior probabilidade de desenvolvimento de lesões agudas e de consequências a longo prazo, ainda que o consumo médio diário não exceda os valores recomendados. ${ }^{2}$

Os dados obtidos revelam um franco aumento do risco de consumo excessivo em MCFA, o que expõe a realidade de excessos generalizada e noticiada pelos media durante as festividades. As razões e as suas eventuais consequências devem ser estudadas.

Quanto à bebida mais consumida num MSFA, 55,9\% dos inquiridos refere a cerveja, $27,8 \%$ outras bebidas (ex.: shots, bebidas espirituosas) e 16,3\% vinho e num MCFA $52,5 \%$ dos inquiridos consome cerveja, 32\% outras (ex.:shots, bebidas espirituosas) e 15,5\% vinho, verificando-se correlação positiva forte e significativa $(p=0,756, p<$ 0,001).

Há diferença significativa entre sexos quanto à bebida mais consumida. A este nível, verifica-se que para o sexo masculino em MCFA, aumenta o consumo preferencial de bebidas espirituosas / shots em detrimento do consumo preferencial de vinho e cerveja, apesar de a cerveja continuar a ser a bebida mais consumida. Para o sexo feminino, não se nota uma alteração do tipo de bebida mais consumida, havendo apenas um ligeiro aumento do consumo preferencial de bebidas espirituosas / shots em detrimento de cerveja, quase a igualando. Em MSFA, a mulher parece preferir bebidas de maior teor alcoólico em relação ao homem, tendência verificada em alunos do Ensino Secundário de Leiria. ${ }^{9}$

Existe diferença significativa entre grupos de frequência do ES quanto à bebida mais consumida em MCFA. O grupo com menor tempo de frequência do ES consome mais bebidas espirituosas e vinho em detrimento da cerveja.

Estes resultados são bastante diferentes dos registados na literatura nacional, que indica que a principal bebida alcoólica consumida é o vinho, seguida da cerveja e de bebidas espirituosas. ${ }^{5,22}$ Contudo, são compatíveis com os resultados de outros estudos efetuados no ES, ${ }^{1}$ em que prevalece o consumo de bebidas fermentadas não espirituosas entre os estudantes, considerando-se mesmo a cerveja como a 'bebida dos estudantes'. É de salientar que o consumo preferencial de bebidas espirituosas é bastante superior nas estudantes de Coimbra quando comparado com outros estudos. 1,4,5,11,12 $^{-1,}$

Ficam por apurar quais os motivos que levam ao consumo preferencial de determinada bebida, o que deverá ser estudado em simultâneo com os consumos superiores nos primeiros anos de ES.

Aponta-se como limitação do estudo a dificuldade de comparação dos resultados, já que diferentes estudos utilizam diferentes ferramentas de 'rastreio' para avaliar os padrões de consumo de álcool, o que é agravado pela escassez de trabalhos publicados em Portugal sobre esta temática. Revela-se, assim, pertinente, alargar e aprofundar a investigação dos padrões de consumo de álcool a outras instituições do ES, possivelmente até ao Ensino Secundário e terceiro ciclo, o que permitirá perceber se o impacto das festas académicas é exclusivo dos estudantes do ES ou se já vem alicerçado em hábitos anteriores. Aparte a existência de festas académicas e o ambiente de desinibição associado, poderá haver características intrínsecas a cada estudante que agora devem ser estudadas.

A identificação da pessoa com consumo excessivo de álcool tem como objetivo a prevenção de patologias associadas. Assim, em futuras investigações, dever-se-á disponibilizar o AUDIT completo àqueles que obtiveram um AUDIT-C positivo, com a respetiva orientação. Para combater este problema, é necessário proceder à mudança de comportamentos. Sabendo-se que $65,8 \%$ dos respondentes reconhecem ter um padrão de consumo diferente com as festas académicas (fase de contemplação?), o próximo passo seria perceber se estariam dispostos a passar à fase seguinte (preparação?).

\section{CONCLUSÃO}

Os níveis de consumo de álcool dos estudantes do Ensino Superior de Coimbra nesta amostra são elevados, com $36,6 \%$ a apresentar risco de consumo excessivo e $40,2 \%$ um consumo superior ao máximo diário recomendado em meses sem festas académicas.

O sexo feminino apresentou maior risco de consumo 
excessivo pelo AUDIT-C.

As festas académicas aumentam o risco de consumo excessivo de bebidas alcoólicas.

O tipo de bebida consumida entre meses sem festas académicas e meses com festas académicas é diferente, registando-se aumento do consumo de bebidas espirituosas/shots por ambos os sexos.

O consumo de bebidas alcoólicas diminui com o tempo de frequência do ES. Contudo, as festas académicas têm um impacto proporcional constante, não associado ao tempo de frequência do ES. No grupo com menor tempo de frequência do ES verifica-se maior consumo de bebidas espirituosas.

\section{AGRADECIMENTOS}

Os autores agradecem à Universidade de Coimbra por disponibilizar a sua página de Facebook ${ }^{\circledR}$ e a todos aqueles que responderam ao inquérito.

\section{CONTRIBUTOS DOS AUTORES}

ACT: Conceção, trabalho de campo, análise de dados, redação do manuscrito.

LMS: Conceção, análise estatística, revisão crítica.

\section{PROTEÇÃO DE PESSOAS E ANIMAIS}

Os autores declaram que os procedimentos seguidos estavam de acordo com os regulamentos estabelecidos pelos responsáveis da Comissão de Investigação Clínica e Ética e de acordo com a Declaração de Helsínquia da Associação Médica Mundial revista em 2013.

\section{CONFIDENCIALIDADE DOS DADOS}

Os autores declaram ter seguido os protocolos do seu centro de trabalho acerca da publicação de dados.

\section{CONFLITO DE INTERESSES}

Os autores declaram não ter qualquer conflito de interesse relativamente ao presente artigo.

\section{FONTES DE FINANCIAMENTO}

Os autores declaram não ter recebido qualquer subsídio ou bolsa relativamente ao presente artigo.

académicas. Porto: Instituto de Ciências Biomédicas de Abel Salazar; 2007.

13. Ferreira A. O consumo de álcool e comportamentos de risco nos estudantes do ensino superior. Aveiro: Universidade de Aveiro; 2008.

14. Costa A, Figueiredo J, Monteiro P, Costa S, Xavier S. Caracterização dos padrões do consumo do álcool em estudantes da universidade de Aveiro. Interacções. 2016;124:112-24.

15. Campos P, Ribeiro C. Rastreio do consumo de álcool nos cuidados de saúde primários - atitudes dos utentes. Rev Port Med Geral Fam. 2012;28:98-104.

16. Cunha J. Validação da versão portuguesa dos Questionários AUDIT e Five-Shot para identificação de consumo excessivo de álcool. Lisboa: Internato Complementar de Clínica Geral da Zona Sul; Instituto de Clínica Geral da Zona Sul; 2002.

17. Fundação Francisco Manuel dos Santos. Base de Dados Portugal Contemporâneo. [consultado 2019 jan 05]. Disponível em: https://www. pordata.pt/DB/Municipios/Ambiente+de+Consulta/Tabela.

18. Reis M, Matos M. Aventura social comportamentos de saúde dos jovens universitários portugueses - Dados Nacionais de 2016. Lisboa: RAventura Social/ FMH/ ULisboa; 2017.

19. Rodrigues P, Salvador A, Lourenço I, Santos L. Padrões de consumo de álcool em estudantes da Universidade de Aveiro: relação com comportamentos de risco e stress. Anal Psicol. 2014;32:453-66.

20. Pedrosa A, Camacho L, Passos S, Oliveira R. Consumo de álcool entre estudantes universitários. Cad Saúde Pública. 2011;27:1611-21.

21. Htet H, Mon Y, Id S, Saw TN, Mie N, Htun M, et al. Prevalence of alcohol consumption and its risk factors among university students : a cross-sectional study across six universities in Myanmar. PLoS One. 2020;15:e0229329.

22. Grácio JC. Determinantes do consumo de bebidas alcoólicas nos estudantes do Ensino Superior de Coimbra. Coimbra: Faculdade de Medicina da Universidadee de Coimbra; 2009. 